\title{
Research on Extraction Process of Gallic Acid from Penthorum chinense Pursh by Aqueous Ethanol
}

\author{
Luping Kang1,2, Shanbin Yang1,2*, Yan Peng1, Jiao Dai' ${ }^{1}$ Xingchun Ying1 \\ ${ }^{1}$ Pharmaceutical Engineering Institute of Engineering Research Center of Biotechnology of Active Materials \\ (Ministry of Education) in Chongqing Normal University, Chongqing, China \\ ${ }^{2}$ Key Laboratory of Green Synthesis and Applications, College of Chemistry, Chongqing Normal University, \\ Chongqing, China \\ Email: shanbiny@126.com
}

Received 19 February 2015; accepted 14 April 2015; published 20 April 2015

Copyright (C) 2015 by authors and Scientific Research Publishing Inc.

This work is licensed under the Creative Commons Attribution International License (CC BY).

http://creativecommons.org/licenses/by/4.0/

(c) (i) Open Access

\begin{abstract}
Penthorum chinense Pursh is rich in gallic acid, which has antioxidant, anti-inflammatory, antifungal and antitumor activities. In order to optimize their extraction conditions, various extraction parameters were chosen to identify their effects on gallic acid extraction. With extraction amount of gallic acid as index, based on single factor analysis, influence of solid/liquid ratio, ethanol concentration, fetch time and extraction temperature on extraction technology were investigated by orthogonal test. The optimization conditions for gallic acid extraction were determined as follows: ethanol concentration $60 \%$, extraction time $2.5 \mathrm{~h}$, temperature $90^{\circ} \mathrm{C}$ and solid/liquid ratio 1:30. The corresponding gallic acid content was $4.85 \%$. This optimized extraction process was stable and feasible.
\end{abstract}

\section{Keywords}

Penthorum, Gallic Acid, Extraction Process, Orthogonal Design

\section{Introduction}

Penthorum chinense Pursh is the species belonging to Saxifragaceae Penthorum perennial herb, and the whole plant is popularly used as medicine in china. It is mainly distributed in Hunan, Sichuan and Guizhou and other provinces. It is a rooted vascular plant, which has the functions of detoxification, diuresis, and promoting blood

*Corresponding author.

How to cite this paper: Kang, L.P., Yang, S.B., Peng, Y., Dai, J. and Ying, X.C. (2015) Research on Extraction Process of Gallic Acid from Penthorum chinense Pursh by Aqueous Ethanol. Green and Sustainable Chemistry, 5, 63-69.

http://dx.doi.org/10.4236/gsc.2015.52009 
circulation. In addition, abundant studies have shown that they have anti-aging, anti-viral, anti-mutation activities [1]. The chemical investigations on $P$. chinense revealed the occurrence of molecules such as flavonoids, gallic acid, sitosterol, organic acid, volatile oil, the major constituents of which are flavonoids and gallic acid [2]. Gallic acid is a hydrolysable tannin monomer, which has the effect of anti-viral and protecting liver [3]-[5]. Extraction flavonoids from $P$. chinense were studied by determining the content of quercetin, quercitrin and gallic acid with HPLC [6] [7]. In this paper, gallic acid from Penthorum chinense pursh extracted with aqueous ethanol was studied. With extraction amount of gallic acid as index, based on single factor analysis, influence of solid/liquid ratio, ethanol concentration, fetch time and extraction temperature on extraction technology was investigated by orthogonal test. The content of gallic acid was determined by ultraviolet-visible spectroscopy. It was conveninent and fast for quality control in actual manufacturing.

\section{Materials and Equipment}

\subsection{Reagents}

Dried Penthorum chinense Pursh was purchased from Gulin Hongan Pharmaceutical Company (Sichuan Province, China). gallic acid (reference standard) was purchased from National Instisutes for Food and Drug Control in China; all the other chemicals used were of analytical grade.

\subsection{General Instrumental Equipment}

An electronic balance was measured on quality (Jingtian electronic instrument company, Shanghai, China, JT202N); An UV-vis spectrophotometer was used for quantification of gallic acid (Analysis of general instrument company, Beijing, China, T6)

\section{Methods and Results}

\subsection{Determination of Gallic Acid Content}

Gallic acid standard substance (25.4 mg) was accurately weighed, dissolved and filled into a $100 \mathrm{~mL}$ volumetric flask. Then $60 \%$ ethanol was added into the volumetric flask. $0.0 \mathrm{~mL}, 1.0 \mathrm{~mL}, 2.0 \mathrm{~mL}, 3.0 \mathrm{~mL}, 4.0 \mathrm{~mL}, 5.0 \mathrm{~mL}$ from $100 \mathrm{~mL}$ volumetric flask was filled into 6 volumetric flasks, respectively. Then $60 \%$ ethanol was added into these volumetric flasks and stood for 10 minutes in dark place. At last, absorbance values were recorded by a T6 spectrophotometer at the wavelength of $279 \mathrm{~nm}$. At the same time, the $60 \%$ ethanol was measured as blank control in an identical way. The regression equation of the gallic acid standard curve was obtained as $\mathrm{A}=$ $50.098 \mathrm{C}+0.1587\left(R^{2}=0.9946\right)$, exhibiting a good linear relationship within the rang of $0.005-0.025 \mathrm{mg} \cdot \mathrm{mL}^{-1}$ (see Figure 1).

\subsection{Gallic Acid Samples and Preparation of Penthorum chinense Pursh Extract}

Penthorum chinense Pursh (6.0 g) was treated by water bath for extraction of gallic acid under the designed conditions. Extraction conditions were adjusted to different ethanol concentrations (30\% - 80\%), ratios of sol$\mathrm{id} /$ liquid $\left(\mathrm{g} \cdot \mathrm{mL}^{-1}\right)(1: 10-1: 35)$, extraction times $(1-3.5 \mathrm{~h})$, and water bath temperatures $\left(50^{\circ} \mathrm{C}-100^{\circ} \mathrm{C}\right)$. When one of the conditions was changed, the other conditions were adjusted accordingly. Penthorum chinense Pursh was extracted twice. After the extracts were combined and filtered, the obtained concentrated solution was dissolved in ethanol (60\%) to $200 \mathrm{~mL}$, then, $2.0 \mathrm{~mL}$ was taken out to a defined volume of $50 \mathrm{~mL}$ for analysis.

\subsection{Precision Experiment}

$3.0 \mathrm{~mL}$ from the standard solution was filled into $50 \mathrm{~mL}$ volumetric falsk, measured 6 times according to the method of $2.1, R S D=1.02 \%$. The precision of this method was good.

\subsection{Stability Test}

$3.0 \mathrm{~mL}$ from the gallic acid sample solution was filled into $50 \mathrm{~mL}$ volumetric falsk every 20 minutes, and measured 6 times according to the method of 2.1, $R S D=1.02 \%$. It showed that the samples were in good stability within 120 minutes. 


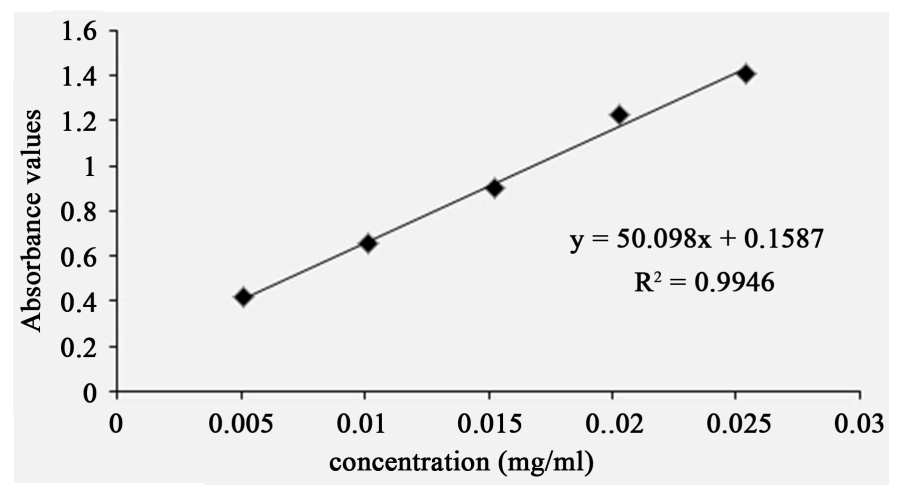

Figure 1. Standard curve of gallic acid.

\subsection{Reproducibility Test}

Six pieces of $P$. chinense ( $2.0 \mathrm{~g}$ ) was treated by water bath for extraction of gallic acid under the designed conditions. The experiment was repeated six times, and measured 6 times according to the method of 2.1, $R S D=$ $1.34 \%$. It showed that the repeatability of the method was good.

\subsection{Recovery Test}

Six pieces of $P$. chinense (6.0 g) were weighed and added a certain amount of gallic acid standard, then measured absorbance values of gallic acid according to the method of 2.1. The experiment was repeated six times, and calculated the recovery of $98.0 \%, R S D=1.42 \%$. It showed that the recovery of the method was good. (see Table 1).

\subsection{Single Factor Results}

\subsubsection{Effect of Solid/Liquid Ratio on Extraction Yield of Gallic Acid from P. chinense}

In this work, the effect of solid/liquid ratio on extraction yield of gallic acid from $P$. chinense was investigated, and the results were listed in Figure 2. Firstly, the other extraction conditions of gallic acid from P. chinense, e.g. extraction temperature, extraction time and ethanol concentration, were fixed at $70^{\circ} \mathrm{C}, 1 \mathrm{~h}, 60 \%$, respectively and solid/liquid ratio was set at 1:10, 1:15, 1:20, 1:25, 1:30, 1:35. As be shown in Figure 2, extraction yield of gallic acid from $P$. chinense continued to increase with solid/liquid ratio and reached the peak value (4.71\%) when solid/liquid ratio was 1:25. The extraction yield of gallic acid from $P$. chinense started to decrease after solid/liquid ratio exceeded 1:25. Therefore, the solid/liquid ratio being set at 1:25 was appropriate.

\subsubsection{Effect of Ethanol Concentration on Extraction Yield of Gallic Acid from P. chinense}

The effect of ethanol concentration on extraction yield of gallic acid from $P$. chinense was shown in Figure 3. Firstly, ethanol concentration was set at $30 \%, 40 \%, 50 \%, 60 \%, 70 \%$, and $80 \%$, while other extraction parameters were given as followings: extraction temperature $70^{\circ} \mathrm{C}$, solid/liquid ratio $1: 20$ and extraction time $1 \mathrm{~h}$. There was similar trend as above for varying solid/liquid ratio on extraction yield of gallic acid from $P$. chinense. With increasing the ethanol concentration from $30 \%$ to $70 \%$, the extraction yield of gallic acid from $P$. chinense first increased and then decreased. The maximum was $4.78 \%$ when the ethanol concentration was $70 \%$ (see Figure 3). Therefore, the ethanol concentration being set at $70 \%$ was appropriate.

\subsubsection{Effect of Extraction Time on Extraction Yield of Gallic Acid from P. chinense}

The effect of extraction time on extraction yield of gallic acid from $P$. chinense was shown in Figure 4. Firstly, extraction time was set at 1, 1.5, 2, 2.5, 3, $3.5 \mathrm{~h}$, while other extraction parameters were given as followings: extraction temperature $70^{\circ} \mathrm{C}$, solid/liquid ratio $1: 20$ and ethanol concentration $60 \%$. It could be found that with increase of extraction time from 1 to $1.5 \mathrm{~h}$, the extraction yield of gallic acid from $P$. chinense increased gradually, and then increased slowly after $1.5 \mathrm{~h}$. Taking everything into account, extraction time being set at $3 \mathrm{~h}$ was appropriate. 


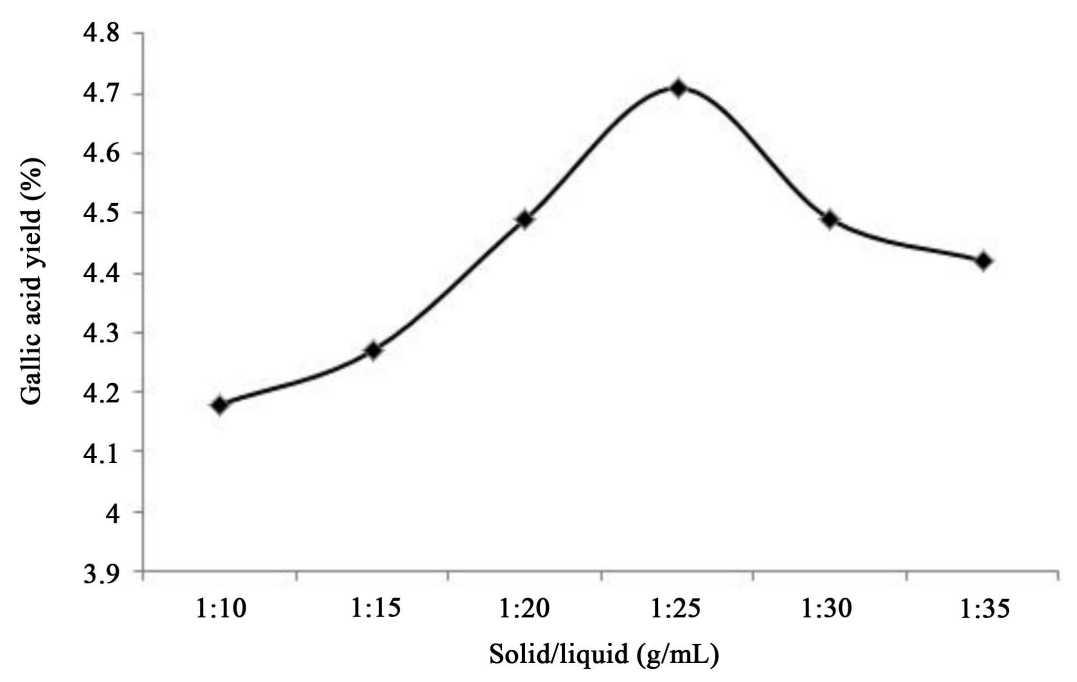

Figure 2. The effect of solid-liquid ratio on extraction rate.

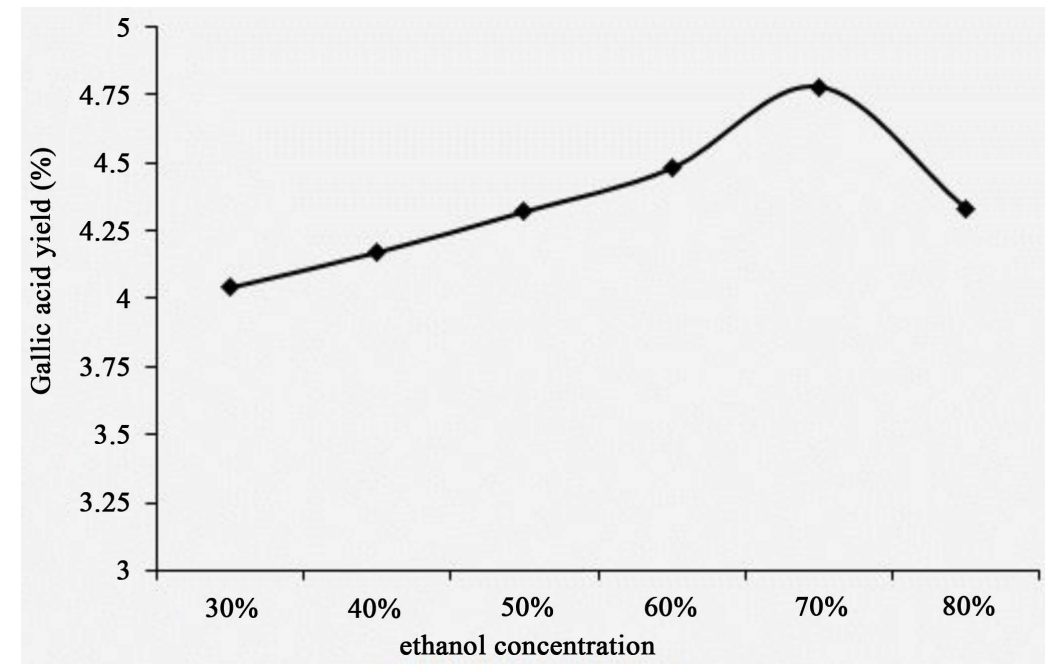

Figure 3. The effect of ethanol concentration on extraction rate.

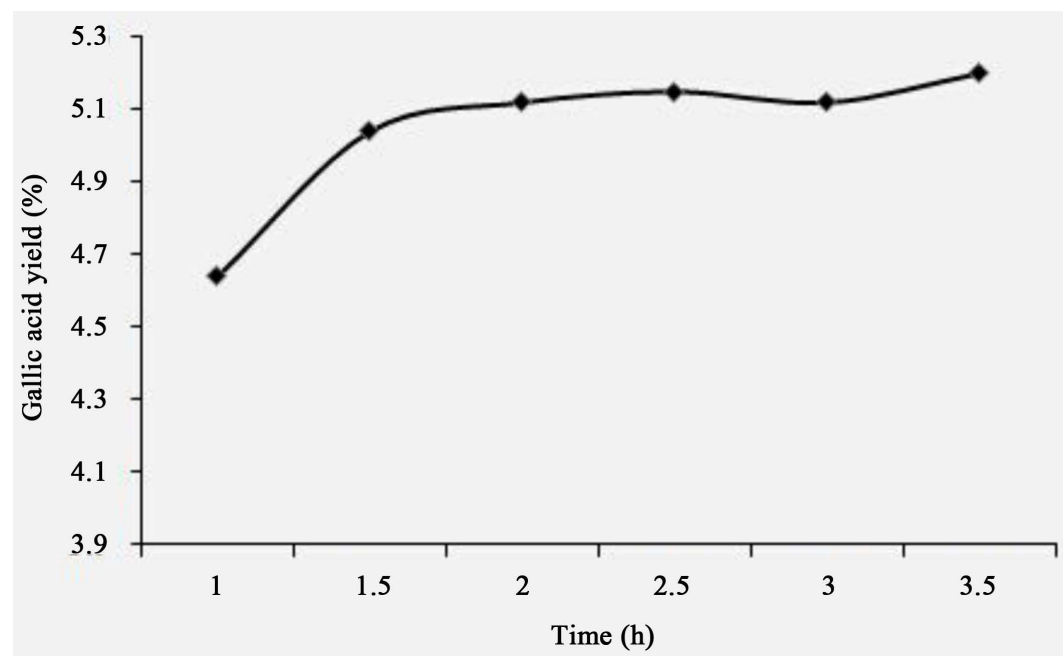

Figure 4. The effect of extraction time on extraction rate. 
Table 1. Result of recovery of gallic acid in Penthorum chinense Pursh.

\begin{tabular}{ccccccc}
\hline No & Original/mg & Added $/ \mathrm{mg}$ & Detected $/ \mathrm{mg}$ & Recovery $\%$ & $\mathrm{X} / \%$ & $\mathrm{RSD} / \%$ \\
1 & 2.7878 & 0.7260 & 3.500 & 98.2 & & \\
2 & 2.7864 & 0.7075 & 3.478 & 97.9 & & \\
3 & 2.7841 & 0.7278 & 3.513 & 100.2 & 98.0 & 1.42 \\
4 & 2.7856 & 0.7131 & 3.490 & 3.463 & 96.8 & \\
5 & 2.7834 & 0.7023 & 3.460 & 96.2 & & \\
6 & 2.7822 & 0.7045 & & & \\
\hline
\end{tabular}

\subsubsection{Effect of Extraction Temperature on Extraction Yield of Gallic acid from P. chinense}

In this work, the effect of different extraction temperature on extraction yield of gallic acid from $P$. chinense was investigated, and the results were listed in Figure 5. Firstly, the other extraction conditions of gallic acid from $P$. chinense, e.g. solid/liquid ratio, extraction time and ethanol concentration, were fixed at 1:20, $1 \mathrm{~h}, 60 \%$, respectively and extraction temperature was set at $50^{\circ} \mathrm{C}, 60^{\circ} \mathrm{C}, 70^{\circ} \mathrm{C}, 80^{\circ} \mathrm{C}, 90^{\circ} \mathrm{C}, 100^{\circ} \mathrm{C}$. As be shown in Figure 5 , extraction yield of gallic acid from $P$. chinense continued to increase as a whole with the increasing extraction temperature. It showed that effect of extraction temperature on extraction yield of gallic acid from $P$. chinense were stable. Due to the boiling point of solvent was close to $80^{\circ} \mathrm{C}$, the extraction temperature being set at $80^{\circ} \mathrm{C}$ was appropriate.

\subsection{Optimization of the Extraction Parameters of Gallic Acid from $P$, chinense}

The first step in the extraction parameters of gallic acid from $P$. chinense was to optimize the operating conditions to obtain an efficient extraction of the target compounds. Since various parameters potentially affected the extraction process, the optimization of the experimental conditions was a critical step in the development of a solvent extraction method. In fact, the extraction temperature, extraction time, solid/liquid ratio and ethanol concentration were generally considered to be the most important factors. Optimization of the suitable extraction conditions in the gallic acid extraction could be carried out by using an experimental design. In the present study, all selected factors were examined using an orthogonal $\mathrm{L}_{9}\left(3^{4}\right)$ test design. The total evaluation index was used to analysis by statistical method. Factors and levels are presented in Table 2. The results of orthogonal test were presented in Table 3. Analysis of variance was presented in Table 4.

The results of experiments presented in Table 3 indicated that the maximum extraction yield of gallic acid was 5.21\%. However, we couldn't select the best extraction conditions only based on these outcomes in Table 3, and a further orthogonal analysis was warranted. Thus, the $\mathrm{K}$ and $\mathrm{R}$ values were calculated and listed in Table 3. As seen from Table 3, we could find that the influence to the mean extraction yields of the compounds decreased in the order: $\mathrm{A}>\mathrm{C}=\mathrm{B}>\mathrm{D}$ according to the $\mathrm{R}$ values. The ethanol concentration was found to be the most important determinant of the yield. In other words, the yield of the gallic acid was obtained when extraction temperature, extraction time, solid/liquid ratio and ethanol concentration were $90^{\circ} \mathrm{C}, 2.5 \mathrm{~h}, 1: 30$ and $60 \%$, respectively. According to Analysis of variance, ethanol concentration was significant in determining a higher extraction yield (Table 4). There were non-significant interaction effects between all other factors.

\subsection{The Best Process Validation Test}

Under the condition of the optimization process, the experiment was repeated six times, and measured the average yield of gallic acid of $4.85 \%, R S D=1.02 \%$. It showed that the process was stable.

\section{Results and Discussion}

Chen [3] reported that four extraction methods of gallic acid were compared by orthogonal test, such as water bath, ultrasonic water bath, decoction, percolation. It determined the optimum condition that extraction temperature, extraction speed, solid/liquid ratio and ethanol concentration were $90^{\circ} \mathrm{C}, 3$ seconds at the rate of 1 drop, 


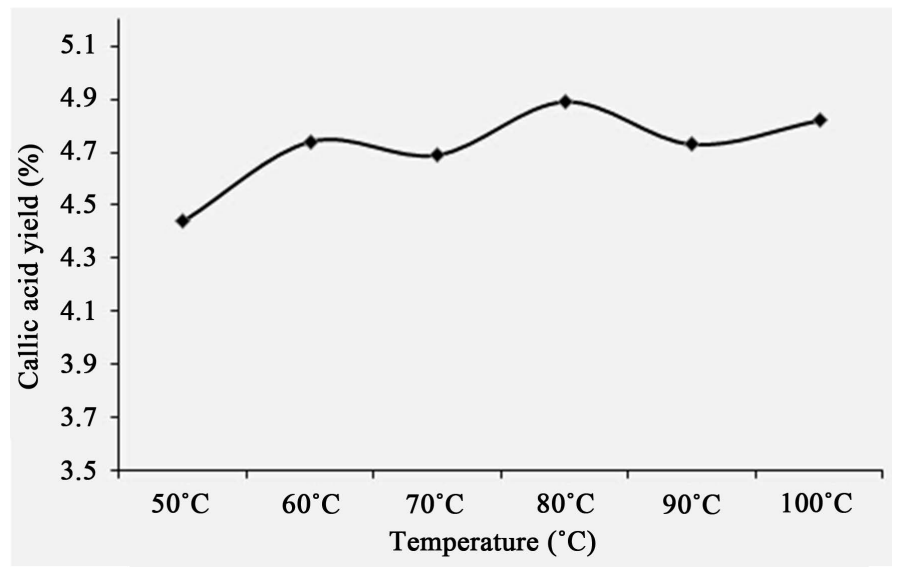

Figure 5. The effect of temperature on extraction rate.

Table 2. Factors and levels of orthogonal test.

\begin{tabular}{ccccc}
\hline Levels & Ethanol concentration (\%) & Solid/liquid ratio & Extraction time $(\mathrm{h})$ & Extraction temperature $\left({ }^{\circ} \mathrm{C}\right)$ \\
\hline & $\mathrm{A}$ & $\mathrm{B}$ & $\mathrm{C}$ & $\mathrm{D}$ \\
1 & $60 \%$ & $1: 20$ & 2.5 & 70 \\
2 & $70 \%$ & $1: 25$ & 3.0 & 80 \\
3 & $80 \%$ & $1: 30$ & 3.5 & 90 \\
\hline
\end{tabular}

Table 3. Results of $\mathrm{L}_{9}\left(3^{4}\right)$ orthogonal test.

\begin{tabular}{cccccc}
\hline Levels & A & B & C & D & Extraction efficiency (\%) \\
\hline 1 & 1 & 1 & 1 & 1 & 5.16 \\
2 & 1 & 2 & 2 & 2 & 5.01 \\
3 & 1 & 3 & 3 & 3 & 5.21 \\
4 & 2 & 1 & 2 & 3 & 5.15 \\
5 & 2 & 2 & 3 & 1 & 5.76 \\
6 & 2 & 3 & 1 & 2 & 4.20 \\
7 & 3 & 1 & 3 & 2 & 4.40 \\
8 & 3 & 2 & 1 & 3 & 4.67 \\
9 & 3 & 3 & 2 & 1 & - \\
I & 15.38 & 14.63 & 14.83 & 14.59 & - \\
II & 15.11 & 14.71 & 14.83 & 14.61 & - \\
III & 13.54 & 15.08 & 14.37 & 14.83 & - \\
R & 1.84 & 0.45 & 0.46 & 0.24 & \\
\hline
\end{tabular}

Table 4. Analysis of variance.

\begin{tabular}{cccccc}
\hline Factors & Sum of squares & F & F value & F critical value & Significant \\
\hline A & 0.674 & 2 & 44.636 & 19 \\
B & 0.059 & 2 & 3.9072 & 19 \\
C & 0.083 & 2 & 5.5333 & 19 \\
D & 0.011 & 2 & 0.7285 & 19 \\
\hline
\end{tabular}

Note: $\mathrm{F}(0.1)=9, \mathrm{~F}(0.05)=19$. 
1:30 and 60\%, respectively. Four methods had their advantages and disadvantages, but the percolation method consumed time longer, was not suitable for the manufacturing. So gallic acid from Penthorum chinense pursh extracted with aqueous ethanol was studied. With extraction amount of gallic acid as index, based on single factor analysis, influence of solid/liquid ratio, ethanol concentration, fetch time and extraction temperature on extraction technology were investigated by orthogonal test. The yield of the gallic acid of $4.85 \%$ was obtained when extraction temperature, extraction time, solid/liquid ratio and ethanol concentration were $90^{\circ} \mathrm{C}, 2.5 \mathrm{~h}, 1: 30$ and $60 \%$, respectively. In this experiment, the aqueous ethanol extraction could shorten extracting time, was more suitable for the industrial production. In the experiment, gallic acid content was determined by simple and fast UV. The method was suitable for quality control in actual manufacturing, and precision, stability, repeatability was good.

\section{Acknowledgements}

This work was supported by the Fund of Doctor's Program in Chongqing Normal University (12XLB006) and the Open Program of Engineering Research Center of Biotechnology of Active Materials (Ministry of Education) in Chongqing Normal University.

In this experiment, our work got these teachers' help (He Daiping, Xiao Feng), thanks!

\section{References}

[1] Xiang, H., Yang, J., Fu, X., et al. (2013) Optimization of Extraction Technology of Penthorum chinense by Orthogonal Test. Chinese Journal of Experimental Traditional Medical Formulae, 19, 50-52.

[2] Chi, S.L., Zhuang, Y.C. and Shui, P.X. (2009) Progress on Penthorum chinense Pursh. Journal of Liaoning University of Traditional Chinese Medicine, 11, 61-64.

[3] Zhang, X. and Yang, M. (2002) Penthorum chinense Pursh Active Ingredients Research. Journal of Chengdu University of Traditional Chinese Medicine, 25, 46-51.

[4] Chen, H. and Li, G. (2009) Study on the Extraction Technology of Gallic Acid from Penthorum chinense by Method of Orthogonal Test Design. Journal of Sichuan of Traditional Chinese Medicine, 27, 56-57.

[5] Zhong, Y.T., Wang, X.L. and Ma, L.L. (2007) Studies on Antimicrobial of Liquidambar Taiwaniana Hance’s Leaf. Lishizhen Medicine and Materia Medica Research, 18, 1693-1694.

[6] Chen, H., Wang, H. and Yuan, H. (2010) Determination of Gallic Acid in Leaf of Liquidambar taiwaniana Hance in Different Areas by HPLC. Chinese Journal of Modern Applied Pharmacy, 27, 840-842.

[7] Lu, Q., Jiang, M.H., Jiang, J.G., et al. (2012) Isolation and Identification of Compounds from Penthorum chinense Pursh with Antioxidant and Antihepatocarcinoma Properties. Journal of Agricultural and Food Chemistry, 60, 1109711103. 